\section{Differentiating AMKL}

\section{By Joanne Kotz, Senior Editor}

A team from the Northwestern University Feinberg School of Medicine and the Broad Institute of MIT and Harvard has shown that inhibiting Aurora kinase A induces cancer cell differentiation in acute megakaryoblastic leukemia, thus reducing tumor load and increasing survival in a mouse model of the disease. ${ }^{1}$ The researchers hope to start clinical trials of Aurora kinase A inhibitors in patients with adult acute megakaryoblastic leukemia next summer.

Acute megakaryoblastic leukemia (AMKL) is a rare form of acute myelogenous leukemia (AML) that is characterized by immature, hyperproliferative megakaryoblasts. Standard of care is chemotherapy.

Megakaryocytes-bone marrow cells that produce plateletstypically develop from hematopoietic stem cells that differentiate to megakaryoblasts and then to mature megakaryocytes. During maturation these cells undergo an unusual process in which the cellular DNA is replicated but the cell does not divide. As a result, mature, platelet-producing megakaryocytes are polyploid-commonly containing as many as 16 sets of chromosomes.

AMKL can be caused by multiple underlying genetic alterations. As a result, targeting any particular alteration would only be expected to provide a therapeutic benefit in a subset of patients.

A team led by John Crispino and Andrew Stern hypothesized that molecules that induced the differentiation of AMKL cells into mature, polyploid megakaryocytes might help take the underlying genetic diversity out of the equation and broadly treat the cancer. Crispino is a professor in the division of hematology/oncology at Northwestern. Stern is associate director of novel therapeutics at the Broad Institute.

"If you look at the genetics that have been associated with AMKL what you see is quite a bit of diversity," said Stern. "The leap here was to ask if there would be a common mechanism that would circumvent the block of differentiation. That's why we took a phenotypic screening approach."

To identify differentiation-inducing small molecules, the team screened a library for compounds that could increase polyploidy in a human AMKL cell line. The group then looked to see which of the hits induced expression of mature megakaryocyte markers and focused its attention on dimethylfasudil, a broad kinase inhibitor.

In all nine AMKL cell lines tested, which represented a variety of the genetic alterations that underlie the disease, dimethylfasudil induced polyploidy and differentiation.

In a mouse model of AMKL, the compound also induced tumor cells in the bone marrow to become polyploid and to differentiate. About 45\% of AMKL mice treated with dimethylfasudil survived at least 70 days, whereas no vehicle-treated mice survived beyond 20 days.
The key question was which kinase was the relevant target for the differentiation-promoting effects of dimethylfasudil. A combination of biochemical, proteomic and genetic approaches all pointed to Aurora kinase A (AURKA; Aurora-A).

Thus, the team turned to alisertib, a selective Aurora-A inhibitor from Takeda Pharmaceutical Co. Ltd.'s Millennium Pharmaceuticals Inc. subsidiary. As predicted, alisertib induced tumor cell polyploidy and decreased disease load compared with vehicle in AMKL mice.

Alisertib (MLN8237) is in Phase III testing in peripheral T cell lymphoma and is in Phase II testing in other hematological malignancies and solid tumors.

Data were published in Cell.

"In my mind the screen for inducers of polyploidization is the most novel and exciting aspect of this work. It has been known for more than two decades that retinoic acid and arsenic induce differentiation in acute promyelocytic leukemia, but these agents were identified empirically, and the mechanism was determined after they were in the clinic," said Ross Levine, an associate member in the Human Oncology \& Pathogenesis Program and a physician in the leukemia service at Memorial SloanKettering Cancer Center.

"I think what was innovative here was to take the approach of looking for a specific process
"In my mind the screen for inducers of polyploidization is the most novel and exciting aspect of this work. It has been known for more than two decades that retinoic acid and arsenic induce differentiation in acute promyelocytic leukemia, but these agents were identified empirically, and the mechanism was determined after they were in the clinic."

-Ross Levine,

Memorial Sloan-Kettering Cancer Center differentiation of these cells. I've not seen that done in this way before," added Neil Thompson, SVP of biology at Astex Pharmaceuticals Inc.

Astex's AT9283, which inhibits multiple kinases including Aurora-A, Aurora-B (AURKB), Janus kinase-2 (JAK-2) and FMS-like tyrosine kinase 3 (FLT3; CD135), is in Phase II testing in multiple myeloma (MM) and in Phase I studies in pediatric leukemia and solid tumors.

\section{Putting differentiation on trial}

The logical next step is testing Aurora-A inhibitors specifically in patients with AMKL.

"The preclinical data in this paper are as good as it gets. I don't think I'd be looking for any additional preclinical studies. They've used the preclinical models that come closest to the clinical situation," said Thompson.

Millennium has completed a Phase II study of alisertib in AML. Liviu Niculescu, senior medical director of global medical affairs at Millennium, said the overall response rate was $13 \%$. 


\section{ANALYSIS}

Niculescu added that no patients with AMKL were treated in the study.

"The outcomes following Aurora-A inhibition in AMKL nonclinical models demonstrated in this paper, polyploidization followed by

\section{"The outcomes following Aurora-A inhibition in AMKL nonclinical models demonstrated in this paper, polyploidization followed by differentiation, provide a strong rationale for testing Aurora-A inhibitors in AMKL patient." \\ -Liviu Niculescu, Millennium Pharmaceuticals Inc.} differentiation, provide a strong rationale for testing Aurora-A inhibitors in AMKL patients," he noted.

Crispino told SciBX that the team is in discussions with Millennium about opening up an adult AMKL-specific trial with alisertib and is in preliminary discussions with two pediatric oncology groups to test Aurora-A inhibitors in patients with pediatric AMKL. Crispino said the team hopes to open the adult AMKL trial next summer.

Millennium would not comment on whether the company is in ongoing discussions, and Niculescu noted that "no plans for an AMKLspecific study have been confirmed at this time."

In addition to Millennium and Astex, at least six other companies have Aurora-A inhibitors in preclinical or clinical development in cancer.

\section{Exploring indications, inhibitors}

The Northwestern-Broad team now is looking at the mechanism of action in additional disease contexts and whether Aurora-A inhibitors with improved activity can be developed.

Myeloproliferative disorders, particularly primary myelofibrosis and essential thrombocytosis, involve megakaryocyte hyperproliferation, noted Crispino. The team therefore wants to test the effects of dimethylfasudil and alisertib in preclinical models of these diseases alone and in combination with Jakafi ruxolitinib.

Jakafi is a JAK-1 and JAK-2 inhibitor marketed by Incyte Corp. and Novartis AG to treat myeloproliferative disorders.

The team also is working to develop new inhibitors. "What we've done is identify Aurora-A as the major target but not necessarily the only target" of dimethylfasudil, said Stern. Thus, Crispino said the group is optimizing dimethylfasudil "with the goal of developing analogs that we could put into Phase I trials."

Northwestern has filed a patent application covering the results reported in the paper. The IP is available for licensing.

Kotz, J. SciBX 5(33); doi:10.1038/scibx.2012.857

Published online Aug. 23, 2012

\section{REFERENCES}

1. Wen, Q. et al. Cell; published online Aug. 3, 2012; doi:10.1016/j.cell.2012.06.032

Contact: John D. Crispino, Northwestern University Feinberg School of Medicine, Chicago, III.

e-mail: j-crispino@northwestern.edu

Contact: Andrew M. Stern, Broad Institute of MIT and Harvard, Cambridge, Mass.

e-mail: astern@broadinstitute.org

\section{COMPANIES AND INSTITUTIONS MENTIONED}

Astex Pharmaceuticals Inc. (NASDAQ:ASTX), Dublin, Calif. Broad Institute of MIT and Harvard, Cambridge, Mass. Incyte Corp. (NASDAQ:INCY), Wilmington, Del. Memorial Sloan-Kettering Cancer Center, New York, N.Y. Millennium Pharmaceuticals Inc. (NASDAQ:MLNM), Cambridge, Mass.

Northwestern University Feinberg School of Medicine, Chicago, III.

Novartis AG (NYSE:NVS; SIX:NOVN), Basel, Switzerland

Takeda Pharmaceutical Co. Ltd. (Tokyo:4502), Osaka, Japan 